Volume 134, Number 2, Pages 491-499

S 0002-9939(05)08037-8

Article electronically published on July 8, 2005

\title{
CUBE-APPROXIMATING BOUNDED WAVELET SETS IN $\mathbb{R}^{n}$
}

\author{
XIAOJIANG YU
}

(Communicated by David R. Larson)

\begin{abstract}
We prove that for any real expansive $n \times n$ matrix $A$, there exists a bounded $A$-dilation wavelet set in the frequency domain $\mathbb{R}^{n}$ (the inverse Fourier transform of whose characteristic function is a band-limited single wavelet in the time domain $\mathbb{R}^{n}$ ). Moreover these wavelet sets can approximate a cube in $\mathbb{R}^{n}$ arbitrarily. This result improves Dai, Larson and Speegle's result about the existence of (basically unbounded) wavelet sets for real expansive matrices.
\end{abstract}

\section{INTRODUCTION}

We say that an $n \times n$ real matrix $A$ is expansive if all the eigenvalues of $A$ have modulus $>1$. We say that an $n \times n$ real matrix $A$ is a strict dilation if $\left\|A^{-1}\right\|<1$ (where $\|\cdot\|$ denotes the matrix norm induced by the Euclidean norm in $\mathbb{R}^{n}$ ). Obviously, a strict dilation must be expansive, but the converse is not true since the spectral radius $\rho\left(A^{-1}\right) \leq\left\|A^{-1}\right\|$ (see $[\mathrm{Br}$, p. 89). In this paper we always suppose that $A$ is real and expansive. An $A$-dilation wavelet basis (abbreviated as wavelets) is a finite collection of measurable functions $\psi_{1}, \ldots, \psi_{m} \in L^{2}\left(\mathbb{R}^{n}\right)$ with the property that

$$
\left\{|\operatorname{det} A|^{\frac{j}{2}} \psi_{i}\left(A^{j} x-k\right): i=1, \ldots, m, j \in \mathbb{Z}, k \in \mathbb{Z}^{n}\right\}
$$

is an orthonormal basis (ONB) of $L^{2}\left(\mathbb{R}^{n}\right)$. The best known method for constructing a wavelet basis is through the use of a multiresolution analysis (MRA). Meyer Me proved that, associated to any MRA, there exist exactly $m:=|\operatorname{det} A|-1$ functions $\psi_{1}, \ldots, \psi_{m}$ which form the wavelet basis. Auscher $\mathrm{Au}$ proved that every wavelet basis whose members satisfy a weak smoothness and decay condition on the Fourier transform side must come from an MRA.

Throughout this paper the Fourier-Plancherel transform and inverse transform are defined as usual:

$$
\begin{gathered}
(\mathcal{F} f)(s):=\int_{\mathbb{R}^{n}} \exp ^{-i(s \cdot t)} f(t) d t, \quad f \in L^{1}\left(\mathbb{R}^{n}\right) \cap L^{2}\left(\mathbb{R}^{n}\right), \\
\left(\mathcal{F}^{-1} g\right)(t)=\frac{1}{(2 \pi)^{n}} \int_{\mathbb{R}^{n}} \exp ^{i(s \cdot t)} g(s) d s, \quad g \in L^{1}\left(\mathbb{R}^{n}\right) \cap L^{2}\left(\mathbb{R}^{n}\right) .
\end{gathered}
$$

Received by the editors March 22, 2004 and, in revised form, September 27, 2004.

2000 Mathematics Subject Classification. Primary 42C15.

Key words and phrases. Real expansive matrix, bounded wavelet set, band-limited wavelet.

The author thanks his supervisor Prof. Jean-Pierre Gabardo for valuable suggestions to revise the primitive results of this paper. The author also thanks Dr. Deguang Han for providing several helpful related preprints. 
Dai, Larson and Speegle [DLS1, DLS2] proved that for any real expansive dilation matrix $A$, there exists a single wavelet associated with it, i.e. a function $\psi \in L^{2}\left(\mathbb{R}^{n}\right)$ such that the collection

$$
\left\{|\operatorname{det} A|^{\frac{j}{2}} \psi\left(A^{j} x-k\right): j \in \mathbb{Z}, k \in \mathbb{Z}^{n}\right\}
$$

is an ONB of $L^{2}\left(\mathbb{R}^{n}\right)$. The single wavelets they constructed have the form $\psi=$ $\mathcal{F}^{-1}\left(\chi_{E}\right)$ for some measurable set $E \subset \mathbb{R}^{n}$ with Lebesgue measure $m(E)=(2 \pi)^{n}$, where $\chi_{E}$ is the characteristic function of $E$. These wavelets are called minimally supported frequency (MSF) wavelets [FW, HWW1, HWW2, and the sets $E$ are called wavelet sets. In light of Auscher's result, it is the lack of smoothness of the Fourier transform of MSF wavelets that makes this possible. The MSF wavelets can be regarded as the generalization of the well-known Shannon wavelet $\mathcal{F}^{-1}\left(\chi_{E}\right)$ in the one-dimensional dyadic setting, where $E=[-2 \pi,-\pi) \cup[\pi, 2 \pi)$.

We can observe that the wavelet sets constructed using the method of Dai, Larson and Speegle are very likely to be unbounded in general. This point was also mentioned in DLS2, p. 18]. In fact, they iteratively used a fact pertaining to dilation-translation pairs, which is equivalent to: given two bounded measurable sets $E, G$ and an open set $F$ in $\mathbb{R}^{n}$, then there exists a pair of $m \in \mathbb{Z}$ and $k \in \mathbb{Z}^{n}$ such that (i) $E+2 k \pi \subset\left(A^{t}\right)^{m} F$, and (ii) $(E+2 k \pi) \cap G \cong \emptyset$. They constructed a disjoint family $\left\{G_{i j}: i \in \mathbb{N}, j \in\{1,2\}\right\}$ of measurable sets whose certain $\left(A^{t}\right)^{m}$ dilates, $m \in \mathbb{Z}$, form a partition $\left\{F_{i j}\right\}$ of $F_{A^{t}}$, a complete wandering set for $A^{t}$, and whose certain $2 \pi k$ translates, $k \in \mathbb{Z}^{n}$, form a partition $\left\{E_{i j}\right\}$ of $E=[-\pi, \pi)^{n}$, modulo Lebesgue null sets. When $G_{i 1}$ are iteratively constructed, they will become unbounded to meet (i) and (ii). Then the wavelet set $G=\bigcup_{i, j} G_{i j}$ will be essentially unbounded.

In this paper we prove the existence of bounded wavelet sets in $\mathbb{R}^{n}$ which can approximate $n$-dimensional cubes for any real expansive dilation matrix $A$, or equivalently, the existence of band-limited MSF wavelets. A noticeable feature of our construction is to leave countably infinitely many components of $E=[-\pi, \pi)^{n}$ unchanged and to translate all the remaining parts of $E$ as a whole by $2 k \pi$ for a certain $k \in \mathbb{Z}^{n}$. Therefore, the wavelet set $G \subset E \cup(E+2 k \pi)$. The construction is concise enough and is actually almost parallel to that in DLS1]. The main difference lies in that an additional property that the point set $\bigcup_{m \in \mathbb{Z}, k \in \mathbb{Z}^{n}} A^{m} k$ is dense in $\mathbb{R}^{n}$ is well used here and was not used in DLS1].

This author has recently noticed a new published paper [DDGH], in which Dai, Diao, $\mathrm{Gu}$ and Han also provided a proof for the existence of bounded wavelet sets for any real expansive dilation matrix. However, their method is more applicable to construct subspace wavelet sets, and MRA or Frame MRA wavelet sets, and hence is much more complicated than our method for the purpose of constructing bounded wavelet sets. Also, their wavelet sets do not have the feature of approximating a cube arbitrarily.

\section{Construction of Wavelet sets}

Throughout this paper we denote $\mathrm{B}_{r}(y)=\left\{x \in \mathbb{R}^{n}:\|x-y\|<r\right\}$, and $\mathrm{B}_{r}:=\mathrm{B}_{r}(0)$. Let $E$ and $F$ be two measurable sets in $\mathbb{R}^{n}$. We say that $E$ and $F$ are $2 \pi$-translation congruent (written as $E \sim_{\tau_{2 \pi}} F$ ) if there are measurable partitions $\left\{E_{p}: p \in \mathbb{Z}\right\}$ and $\left\{F_{p}: p \in \mathbb{Z}\right\}$ of $E$ and $F$, respectively, and a sequence $\left\{n_{p}: p \in \mathbb{Z}\right\} \subset \mathbb{Z}^{n}$ such that $E_{p}=F_{p}+2 \pi n_{p}$ for each $p \in \mathbb{Z}$, modulo null sets; then 
$m(E)=m(F)$ is implied. We say that $E$ and $F$ are $A$-dilation congruent (written as $E \sim_{\delta_{A}} F$ ) if there are measurable partitions $\left\{E_{p}: p \in \mathbb{Z}\right\}$ and $\left\{F_{p}: p \in \mathbb{Z}\right\}$ of $E$ and $F$, respectively, and a sequence $\left\{l_{p}: p \in \mathbb{Z}\right\} \subset \mathbb{Z}$ such that $E_{p}=A^{l_{p}} F_{p}$ for each $p \in \mathbb{Z}$, modulo null sets (see [DLS1, GH]). Dilation congruence does not preserve measure. A measurable set with finite measure can even be $A$-dilation congruent to another measurable set with infinite measure.

The following lemma states a fact which might already be well known and was even used in DLS1 for instance. However, no proof is given in DLS1, and the proof for it cannot be found in many textbooks on matrix analysis either. Because of its important role in the theory of wavelet sets, we will provide a proof for it here for the readers' convenience.

Lemma 2.1. Let $A$ be a real expansive matrix, that is, all the eigenvalues of $A$ have modulus $>1$. Then, $A$ is similar to a real strict dilation matrix $C$. That is, $A=T^{-1} C T$ for two real invertible matrices $T$ and $C$ with $\left\|C^{-1}\right\|<1$.

Proof. We will take two steps to prove this lemma. In step one we first prove that $A$ is similar to a complex strict dilation matrix $C_{0}$. That is, $A=T_{0}^{-1} C_{0} T_{0}$ for two complex invertible matrices $T_{0}$ and $C_{0}$ with $\left\|C_{0}^{-1}\right\|<1$. Suppose $A^{-1}=U^{-1} \Lambda U$, where $U$ is a complex invertible matrix, and $\Lambda$ is the Jordan canonical form of $A^{-1}$. We will choose another complex matrix $V$ such that $V \Lambda V^{-1}=C_{0}^{-1}$ with $\left\|C_{0}^{-1}\right\|<1$. Let $T_{0}=V U$; then $A=T_{0}^{-1} C_{0} T_{0}$. For simplicity, we will take $V$ to be diagonal. Since $\left\|C_{0}^{-1}\right\|=\left\{\rho\left(\left(C_{0}^{-1}\right)^{*} C_{0}^{-1}\right)\right\}^{\frac{1}{2}}$, where $\rho$ and $*$ denote the spectral radius and the complex conjugate transpose, respectively, it suffices that we prove this statement in the case where $\Lambda$ is simply a Jordan block. Let

$$
\Lambda=\left(\begin{array}{ccccc}
\lambda & & & & \\
1 & \lambda & & & \\
& 1 & \lambda & & \\
& & \ddots & \ddots & \\
& & & 1 & \lambda
\end{array}\right)_{k \times k}
$$

be a $k \times k$ Jordan block with $|\lambda|<1, V=\operatorname{diag}\left(v_{1}, \ldots, v_{k}\right)$, and $C_{0}^{-1}=V \Lambda V^{-1}$. Then

$$
\begin{aligned}
& \left(C_{0}^{-1}\right)^{*} C_{0}^{-1}
\end{aligned}
$$

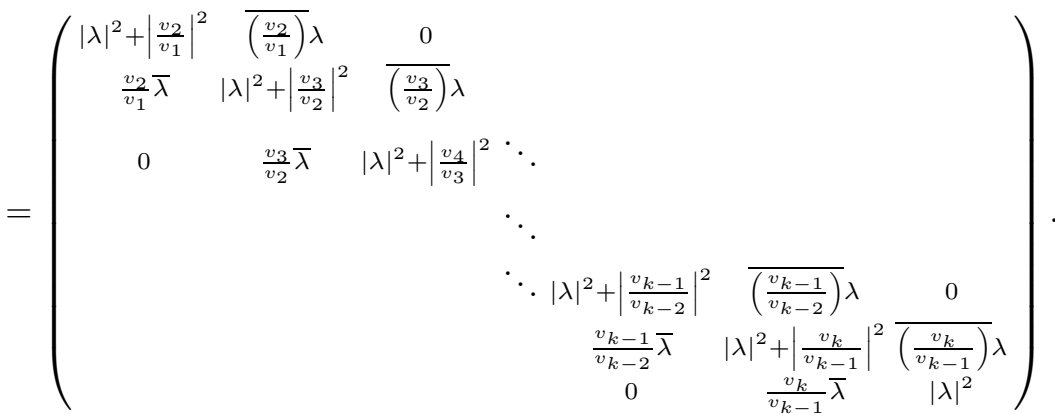

This matrix is tridiagonal. Fix $v_{1} \neq 0$. Since $|\lambda|<1$, we may choose $v_{2} \neq 0$ such that the sum of the moduli of the first row is less than one. For the chosen fixed $v_{2}$, we may choose $v_{3} \neq 0$ such that the sum of the moduli of the second row is less than one. We proceed in this way until $v_{k} \neq 0$ is chosen such that the sum of the 
moduli of the $(k-1)$-th row and that of the $k$-th row are less than one, respectively. By the Gerschgorin's Circle Theorem, the spectral radius of $\left(C_{0}^{-1}\right)^{*} C_{0}^{-1}$ is less than one. Thus $\left\|C_{0}^{-1}\right\|<1$.

In step two, we prove that $A$ is similar to a real strict dilation matrix. Write $T_{0}=\operatorname{Re}\left(T_{0}\right)+i \operatorname{Im}\left(T_{0}\right)$. Then

$$
\operatorname{Re}\left(T_{0}^{*} T_{0}\right)=\left(\operatorname{Re}\left(T_{0}\right)\right)^{t} \operatorname{Re}\left(T_{0}\right)+\left(\operatorname{Im}\left(T_{0}\right)\right)^{t} \operatorname{Im}\left(T_{0}\right)
$$

and

$$
x^{t} \operatorname{Re}\left(T_{0}^{*} T_{0}\right) x=\left|\operatorname{Re}\left(T_{0}\right) x\right|^{2}+\left|\operatorname{Im}\left(T_{0}\right) x\right|^{2} \geq 0 \quad \text { for } x \in \mathbb{R}^{n} .
$$

If $x^{t} \operatorname{Re}\left(T_{0}^{*} T_{0}\right) x=0$ for some $x \neq 0$, then $T_{0} x=\operatorname{Re}\left(T_{0}\right) x+i \operatorname{Im}\left(T_{0}\right) x=0$ for this $x$. This is impossible since $T_{0}$ is invertible. $\operatorname{So} \operatorname{Re}\left(T_{0}^{*} T_{0}\right)$ is real symmetric and positive definite. Let $\mathcal{B}_{1}$ and $\overline{\mathcal{B}_{1}}$ denote the open and closed unit balls centered at the origin 0 in the complex $n$-dimensional space $\mathbb{C}^{n}$, respectively. Since $A=T_{0}^{-1} C_{0} T_{0}$ and $\left\|C_{0}^{-1}\right\|<1$, it follows that $\overline{\mathcal{B}_{1}} \subsetneq C_{0} \mathcal{B}_{1}$, and $T_{0}^{-1} \overline{\mathcal{B}_{1}} \subsetneq A\left(T_{0}^{-1} \mathcal{B}_{1}\right)$. Define $F=\left\{z \in \mathbb{C}^{n}: z \in T_{0}^{-1} \mathcal{B}_{1}\right.$ and $\left.\operatorname{Im}(z)=0\right\}$. Then

$$
\begin{aligned}
F & =T_{0}^{-1} \mathcal{B}_{1} \cap \mathbb{R}^{n}=\left\{x \in \mathbb{R}^{n}: x^{t} T_{0}^{*} T_{0} x<1\right\} \\
& =\left\{x \in \mathbb{R}^{n}: x^{t} \operatorname{Re}\left(T_{0}^{*} T_{0}\right) x<1\right\}
\end{aligned}
$$

is a real open neighborhood of 0 in $\mathbb{R}^{n}$. Note that for every $x \in \mathbb{R}^{n}, x^{t} \operatorname{Im}\left(T_{0}^{*} T_{0}\right) x=$ 0 . Let $\bar{F}$ denote the closure of $F$ in $\mathbb{R}^{n}$. Then accordingly,

$$
\begin{aligned}
\bar{F} & =\left\{x \in \mathbb{R}^{n}: x^{t} \operatorname{Re}\left(T_{0}^{*} T_{0}\right) x \leq 1\right\} \\
& =\left\{x \in \mathbb{R}^{n}: x^{t} T_{0}^{*} T_{0} x \leq 1\right\}=T_{0}^{-1} \overline{\mathcal{B}_{1}} \cap \mathbb{R}^{n} \\
& \subsetneq A\left(T_{0}^{-1} \mathcal{B}_{1}\right) \cap \mathbb{R}^{n}=A F .
\end{aligned}
$$

Since $\operatorname{Re}\left(T_{0}^{*} T_{0}\right)$ is symmetric and positive definite, we write $\operatorname{Re}\left(T_{0}^{*} T_{0}\right)=T^{2}$, where $T$ is the unique symmetric positive definite square root matrix of $\operatorname{Re}\left(T_{0}^{*} T_{0}\right)$. Then, by (2.1), $F=\left\{x \in \mathbb{R}^{n}:\|T x\|<1\right\}=T^{-1} \mathrm{~B}_{1}$ and $\bar{F}=T^{-1} \overline{\mathrm{B}}_{1}$. Therefore, $T A^{-1} T^{-1} \overline{\mathrm{B}_{1}} \subsetneq \mathrm{B}_{1}$ implies $\left\|T A^{-1} T^{-1}\right\|<1$.

If $A$ is a strict dilation, so $\left\|A^{-1}\right\|<1$, then $A \mathrm{~B}_{1} \supseteq \mathrm{B}_{\left\|A^{-1}\right\|^{-1}} \supsetneq \mathrm{B}_{1}$. It follows that, if $F_{A}=A \mathrm{~B}_{1} \backslash \mathrm{B}_{1}$, then $\left\{A^{k} F_{A}: k \in \mathbb{Z}\right\}$ is a partition of $\mathbb{R}^{n} \backslash\{0\}$. If $A$ is expansive, then $A=T^{-1} C T$ for two real invertible matrices $T$ and $C$ with $\left\|C^{-1}\right\|<1$. If $F_{A}=T^{-1}\left(C \mathrm{~B}_{1} \backslash \mathrm{B}_{1}\right)$, then $\left\{A^{k} F_{A}: k \in \mathbb{Z}\right\}$ is a partition of $\mathbb{R}^{n} \backslash\{0\}$. Therefore, an expansive matrix $A$ always has a measurable complete wandering set $F_{A} \subset \mathbb{R}^{n}$. That is, $L^{2}\left(\mathbb{R}^{n}\right)$ is the direct sum decomposition of the subspaces $\left\{D_{A}^{k} L^{2}\left(F_{A}\right)\right\}_{k \in \mathbb{Z}}$, where $D_{A}$ is the unitary operator defined by

$$
\left(D_{A} f\right)(x)=|\operatorname{det} A|^{\frac{1}{2}} f(A x), \quad \text { for } f \in L^{2}\left(\mathbb{R}^{n}\right), x \in \mathbb{R}^{n} .
$$

It is clear that any measurable set $F^{\prime}$ with $F^{\prime} \sim_{\delta_{A}} F_{A}$ has the same property.

Remark 2.1. Suppose $E_{A}$ is an open bounded neighborhood of 0 such that $E_{A} \subset$ $A E_{A}$ and $F_{A}=A E_{A} \backslash E_{A}$ has nonempty interior. If $M$ is an $n \times n$ real matrix which commutes with $A$, for instance $M=r A^{j}$ for $r>0, j \in \mathbb{Z}$, then $M E_{A}$ is still an open bounded neighborhood of 0 such that $M E_{A} \subset A M E_{A}$ and $A M E_{A} \backslash M E_{A}=M F_{A}$ has nonempty interior.

The following characterization of wavelet sets, whose proof can be seen in DLS1] and $[\mathrm{GH}]$, will play a major role in this paper. 
Proposition 2.2. A measurable set $W \subset \mathbb{R}^{n}$ is an $A$-dilation wavelet set if and only if $W$ is both $A^{t}$-dilation congruent to $F_{A^{t}}$, a complete wandering set for $A^{t}$, and $2 \pi$-translation congruent to $E=[-\pi, \pi)^{n}$.

The main result of this paper is the following theorem on dual-dynamical system congruency. The existence of bounded wavelet sets is a corollary of it.

Theorem 2.3. Let $A$ be an $n \times n$ real expansive matrix. Suppose $E$ and $F$ are two bounded measurable sets in $\mathbb{R}^{n}$ such that $E$ contains a neighborhood of 0 , and $F$ has nonempty interior and $F$ is bounded away from 0 . Then there is a $k \in \mathbb{Z}^{n} \backslash\{0\}$ satisfying the following properties: $E \cap(E+2 \pi k) \cong \emptyset$, and given any small $\epsilon>0$ with $\mathrm{B}_{\epsilon} \subset E$, we can construct two measurable sets of positive measure $G_{I}$ and $G_{I I}$ with $G_{I} \subset \mathrm{B}_{\epsilon} \subset E$ and $G_{I I} \subset E+2 \pi k$ such that $G=G_{I} \cup G_{I I}$ is both A-dilation congruent to $F$ and $2 \pi$-translation congruent to $E$.

Proof. We first claim that we can choose a point $c \in F^{\circ}$, the interior of $F$, an integer $m \in \mathbb{Z}$, an integer vector $k \in \mathbb{Z}^{n} \backslash\{0\}$, and a positive number $\alpha_{1}>0$ such that

$$
\text { (i) } A^{-m} E \subset \mathrm{B}_{\alpha_{1}} \text {, (ii) } c=2 \pi A^{-m} k \text {, (iii) } E \cap(E+2 \pi k) \cong \emptyset \text {, }
$$$$
\text { (iv) } A^{-m}(E+2 k \pi) \subset \mathrm{B}_{\alpha_{1}}(c) \subset F \text {, (v) } F \backslash \mathrm{B}_{\alpha_{1}}(c) \text { has positive measure. }
$$

Proof of the claim. Pick a point $c_{0} \neq 0$ in the interior of $F$ such that $\mathrm{B}_{\alpha}\left(c_{0}\right) \subset F$ for some $\alpha>0$. Let $\alpha_{1}=\alpha / 2$. Since $A$ is expansive then $\left\|A^{-m}\right\| \rightarrow 0$ as $m \rightarrow+\infty$ (this is implied by Lemma 2.1, or see [Br, pp. 92-93]). So when $m$ is sufficiently large we have

$$
\text { (vi) } A^{-m}\left(E \cup[0,2 \pi)^{n}\right) \subset \mathrm{B}_{\alpha_{1}} \text {. }
$$

Note that for any $m \in \mathbb{Z}$ there is a unique $k_{m} \in \mathbb{Z}^{n}$ such that $k_{m}-\frac{1}{2 \pi} A^{m} c_{0} \in[0,1)^{n}$. Write $c_{m}=\frac{1}{2 \pi} A^{m} c_{0}$. Since $0<\left\|\frac{1}{2 \pi} c_{0}\right\| \leq\left\|A^{-m}\right\|\left\|c_{m}\right\|$, the sequence $\left\{\left\|c_{m}\right\|\right\}$ must be unbounded as $m \rightarrow+\infty$, and thus $\left\{\left\|k_{m}\right\|\right\}$ is unbounded as $m \rightarrow+\infty$, since $k_{m}-c_{m} \in[0,1)^{n}$. Therefore, we can choose a positive integer $m \in \mathbb{N}$ large enough such that (iii) and (vi) both hold. With this chosen $m$ fixed, we define $k=k_{m}$ and $c=2 \pi A^{-m} k$. Then

$$
c-c_{0}=2 \pi A^{-m}\left(k_{m}-\frac{1}{2 \pi} A^{m} c_{0}\right) \in 2 \pi A^{-m}[0,1)^{n} \subset \mathrm{B}_{\alpha_{1}}=\mathrm{B}_{\alpha_{1}}\left(c_{0}\right)-c_{0} .
$$

This means that $c \in \mathrm{B}_{\alpha_{1}}\left(c_{0}\right)$. Also, we have the inclusion $\mathrm{B}_{\alpha_{1}}(c) \subset \mathrm{B}_{\alpha}\left(c_{0}\right) \subset F$, and know that $F \backslash \mathrm{B}_{\alpha_{1}}(c)$ has positive measure since $\alpha_{1}=\alpha / 2$. The first inclusion in (iv) is implied by (i) and (ii). This completes the proof of the claim.

Now give any $\epsilon>0$ such that $\mathrm{B}_{\epsilon} \subset E$. We construct inductively a disjoint family $\left\{G_{i j}: i \in \mathbb{N}, j=1,2\right\}$ of measurable sets whose certain $A^{p}$-dilates, $p \in \mathbb{Z}$, form a partition $\left\{F_{i j}\right\}$ of $F$ and whose certain $2 \pi q$-translates, $q \in \mathbb{Z}^{n}$, form a partition $\left\{E_{i j}\right\}$ of $E$, modulo null sets, such that $G_{I}=\bigcup_{i} G_{i 1} \subset \mathrm{B}_{\epsilon}$ and $G_{I I}=\bigcup_{i} G_{i 2} \subset$ $E+2 \pi k$. For the first step, let $F_{11}=F \backslash \mathrm{B}_{\alpha_{1}}(c)$. Choose $l_{1} \in \mathbb{Z}$ such that $A^{l_{1}} F_{11} \subset \mathrm{B}_{\epsilon} \subset E$. Let $E_{11}=G_{11}=A^{l_{1}} F_{11}$. This set has positive measure, and is bounded away from 0 since $F$ is. Fix $\beta_{1}$ such that $0<\beta_{1}=\frac{1}{2} \inf \left\{\|x\|: x \in E_{11}\right\}$ implying $\mathrm{B}_{\beta_{1}} \subset \mathrm{B}_{\epsilon}$. Fix $\alpha_{2}$ with $0<\alpha_{2}=\frac{1}{2} \inf \left\{\left\|A^{-m} x\right\|: x \in E \backslash \mathrm{B}_{\beta_{1}}\right\}<\frac{1}{2} \alpha_{1}$ by (i). Note that the set $\left\{x \in \mathbb{R}^{n}:\left\|A^{-m} x\right\|=\alpha_{2}\right\}$ is completely contained in $\mathrm{B}_{\beta_{1}}$ and is bounded away from 0 , since $\mathbb{R}^{n}: x \longrightarrow A^{-m} x$ is a homeomorphism. Write 
$V_{1}=\left\{x \in \mathbb{R}^{n}:\left\|A^{-m} x\right\|<\alpha_{2}\right\}$. Let $E_{12}=E \backslash\left(E_{11} \cup V_{1}\right)$, which has positive measure by the choice of $\alpha_{2}$. Also, let $G_{12}=E_{12}+2 k \pi$ and $F_{12}=A^{-m} G_{12}$. Then $F_{12} \subset \mathrm{B}_{\alpha_{1}}(c) \backslash \mathrm{B}_{\alpha_{2}}(c)$ by (iv) and the construction of $V_{1}$. For the second step, let

$$
F_{21}=\mathrm{B}_{\alpha_{1}}(c) \backslash\left(\mathrm{B}_{\alpha_{2}}(c) \cup F_{12}\right)=\left(\left(\mathrm{B}_{\alpha_{1}} \backslash A^{-m} E\right) \cup A^{-m} E_{11}\right)+c,
$$

which has positive measure. Choose $l_{2} \in \mathbb{Z}$ such that $A^{l_{2}} F_{21} \subset V_{1}$. Let $E_{21}=$ $G_{21}=A^{l_{2}} F_{21}$, and notice that this set is bounded away from 0 . Fix $\beta_{2}$ with $0<\beta_{2}=\frac{1}{2} \inf \left\{\|x\|: x \in E_{21} \cup \partial V_{1}\right\}<\frac{1}{2} \beta_{1}$, where $\partial V_{1}$ is the boundary of $V_{1}$. Fix $\alpha_{3}$ with $0<\alpha_{3}=\frac{1}{2} \inf \left\{\left\|A^{-m} x\right\|: \quad x \in V_{1} \backslash \mathrm{B}_{\beta_{2}}\right\}<\frac{1}{2} \alpha_{2}$. Then the set $\left\{x \in \mathbb{R}^{n}:\left\|A^{-m} x\right\|=\alpha_{3}\right\}$ is completely contained in $\mathrm{B}_{\beta_{2}}$ and is bounded away from 0. Write $V_{2}=\left\{x \in \mathbb{R}^{n}:\left\|A^{-m} x\right\|<\alpha_{3}\right\}$. Let $E_{22}=V_{1} \backslash\left(E_{21} \cup V_{2}\right)$, $G_{22}=E_{22}+2 k \pi$, and $F_{22}=A^{-m} G_{22}$. Then $F_{22} \subset \mathrm{B}_{\alpha_{2}}(c) \backslash \mathrm{B}_{\alpha_{3}}(c)$ by the construction of $V_{1}$ and $V_{2}$. For the third step, let

$$
F_{31}=\mathrm{B}_{\alpha_{2}}(c) \backslash\left(\mathrm{B}_{\alpha_{3}}(c) \cup F_{22}\right)=A^{-m}\left(G_{21}+2 \pi k\right)
$$

and so on.

Proceeding inductively, we obtain disjoint families of measurable sets $\left\{E_{i j}\right\} \subset E$, $\left\{F_{i j}\right\} \subset F$, and $\left\{G_{i j}\right\}$ such that

$$
\begin{aligned}
& G_{i 1}=E_{i 1}, \quad G_{i 2}=E_{i 2}+2 k \pi, \quad i=1,2, \ldots, \\
& G_{i 1}=A^{l_{i}} F_{i 1}, \quad G_{i 2}=A^{m} F_{i 2}, \quad i=1,2, \ldots, \\
& \text { (and also } \left.F_{i 1}=A^{-m}\left(G_{i-1,1}+2 \pi k\right), \quad i=3,4, \ldots\right) .
\end{aligned}
$$

We have $E \backslash\left(\bigcup_{i, j} E_{i j}\right)=\{0\}$, a null set, and $F \backslash\left(\bigcup_{i, j} F_{i j}\right)=\{c\}$, a null set, since our procedure makes both $\alpha_{i}$ and $\beta_{i}$ go to zero as $i \rightarrow \infty$. Then

$$
G=\bigcup_{i, j} G_{i j}=\left(\bigcup_{i} E_{i 1}\right) \cup\left(\bigcup_{i} E_{i 2}+2 k \pi\right) \subset \mathrm{B}_{\epsilon} \cup(E+2 k \pi)
$$

is bounded. This finishes the proof.

Corresponding to Dai, Larson and Speegle's remark [DLS1] that an infinite pairwise disjoint family $\left\{G^{k}\right\}_{k=1}^{\infty}$ of wavelet sets can be constructed, now we briefly describe how to construct a countably infinite pairwise essentially disjoint family of bounded measurable sets, $\left\{G^{(r)}\right\}_{r=1}^{\infty}$, each of which is both $A$-dilation congruent to $F$ and $2 \pi$-translation congruent to $E$. The construction procedure resembles the well-known diagonal rule for selecting a subsequence. Choose the same $c_{0}$ in the interior of $F$ such that $\mathrm{B}_{\alpha}\left(c_{0}\right) \subset F$ for some $\alpha>0$ as in the proof of Theorem 2.3. Let $\alpha_{1}^{(r)}=\frac{\alpha}{2}$ for $r=1,2, \ldots$ We can choose countably infinite triples $\left\{c^{(r)}, m^{(r)}, k^{(r)}\right\}_{r \in \mathbb{N}}$ such that each triple satisfies the five conditions in (2.2) and such that the sets $E+2 \pi k^{(r)}, r \in \mathbb{N}$, are pairwise essentially disjoint. Note that all these triples are chosen before we start to construct each $G^{(r)}$. The structure of each $G^{(r)}=\bigcup_{i, j} G_{i j}^{(r)}$ is similar to that of $G$ in the proof of Theorem 2.3. Obviously, $G_{i 2}^{(r)} \cap G_{j 2}^{(s)} \cong \emptyset$ if $r \neq s, i, j \in \mathbb{N}$, by the choice of $k^{(r)}$ and (2.3). To ensure that all the sets $G_{i 1}^{(r)}=E_{i 1}^{(r)}, i, r \in \mathbb{N}$, are pairwise essentially disjoint, we have to construct them according to the following steps. In these steps each sign $\Longrightarrow$ does not mean 
implying or leading to, but means from the current step going or turning to the next step.

$$
\begin{aligned}
& F_{11}^{(1)}=F \backslash \mathrm{B}_{\alpha_{1}^{(1)}}\left(c^{(1)}\right), G_{11}^{(1)}=A^{l_{1}^{(1)}} F_{11}^{(1)} \subset \mathrm{B}_{\epsilon}, F_{11}^{(2)}=F \backslash \mathrm{B}_{\alpha_{1}^{(2)}}\left(c^{(2)}\right), \\
& G_{11}^{(2)}=A_{1}^{l_{1}^{(2)}} F_{11}^{(2)} \subset \mathrm{B}_{\epsilon} \backslash G_{11}^{(1)}, \beta_{1}^{(1)}=\frac{1}{2} \inf \left\{\|x\|: x \in G_{11}^{(1)} \cup G_{11}^{(2)}\right\}, \\
& \alpha_{2}^{(1)}=\frac{1}{2} \inf \left\{\left\|A^{-m^{(1)}} x\right\|: x \in E \backslash \mathrm{B}_{\beta_{1}^{(1)}}\right\}, V_{1}^{(1)}=\left\{x \in \mathbb{R}^{n}:\left\|A^{-m^{(1)}} x\right\|<\alpha_{2}^{(1)}\right\}, \\
& G_{12}^{(1)}=E \backslash\left(G_{11}^{(1)} \cup V_{1}^{(1)}\right)+2 \pi k^{(1)}, F_{12}^{(1)}=A^{-m^{(1)}} G_{12}^{(1)}, \\
& F_{21}^{(1)}=\mathrm{B}_{\alpha_{1}^{(1)}}\left(c^{(1)}\right) \backslash\left(\mathrm{B}_{\alpha_{2}^{(1)}}\left(c^{(1)}\right) \cup F_{12}^{(1)}\right), G_{21}^{(1)}=A_{2}^{l_{2}^{(1)}} F_{21}^{(1)} \subset V_{1}^{(1)}, \\
& F_{11}^{(3)}=F \backslash \mathrm{B}_{\alpha_{1}^{(3)}}\left(c^{(3)}\right), G_{11}^{(3)}=A_{1}^{l_{1}^{(3)}} F_{11}^{(3)} \subset V_{1}^{(1)} \backslash G_{21}^{(1)}, \\
& \beta_{1}^{(2)}=\frac{1}{2} \inf \left\{\|x\|: x \in G_{21}^{(1)} \cup G_{11}^{(3)} \cup \partial V_{1}^{(1)}\right\}, \\
& \alpha_{2}^{(2)}=\frac{1}{2} \inf \left\{\left\|A^{-m^{(2)}} x\right\|: x \in E \backslash \mathrm{B}_{\beta_{1}^{(2)}}\right\}, V_{1}^{(2)}=\left\{x \in \mathbb{R}^{n}:\left\|A^{-m^{(2)}} x\right\|<\alpha_{2}^{(2)}\right\}, \\
& G_{12}^{(2)}=E \backslash\left(G_{11}^{(2)} \cup V_{1}^{(2)}\right)+2 \pi k^{(2)}, F_{12}^{(2)}=A^{-m^{(2)}} G_{12}^{(2)}, \\
& F_{21}^{(2)}=\mathrm{B}_{\alpha_{1}^{(2)}}\left(c^{(2)}\right) \backslash\left(\mathrm{B}_{\alpha_{2}^{(2)}}\left(c^{(2)}\right) \cup F_{12}^{(2)}\right), G_{21}^{(2)}=A^{l_{2}^{(2)}} F_{21}^{(2)} \subset V_{1}^{(2)}, \\
& \beta_{2}^{(1)}=\frac{1}{2} \inf \left\{\|x\|: x \in G_{21}^{(2)} \cup \partial V_{1}^{(2)}\right\}, \alpha_{3}^{(1)}=\frac{1}{2} \inf \left\{\left\|A^{-m^{(1)}} x\right\|: x \in V_{1}^{(1)} \backslash \mathrm{B}_{\beta_{2}^{(1)}}\right\}, \\
& V_{2}^{(1)}=\left\{x \in \mathbb{R}^{n}:\left\|A^{-m^{(1)}} x\right\|<\alpha_{3}^{(1)}\right\}, G_{22}^{(1)}=V_{1}^{(1)} \backslash\left(G_{21}^{(1)} \cup V_{2}^{(1)}\right)+2 \pi k^{(1)}, \\
& F_{22}^{(1)}=A^{-m^{(1)}} G_{22}^{(1)}, F_{31}^{(1)}=\mathrm{B}_{\alpha_{2}^{(1)}}\left(c^{(1)}\right) \backslash\left(\mathrm{B}_{\alpha_{3}^{(1)}}^{(1)}\left(c^{(1)}\right) \cup F_{22}^{(1)}\right), \\
& G_{31}^{(1)}=A^{l_{3}^{(1)}} F_{31}^{(1)} \subset V_{2}^{(1)}, F_{11}^{(4)}=F \backslash \mathrm{B}_{\alpha_{1}^{(4)}}\left(c^{(4)}\right), \\
& {\left[G_{11}^{(4)}=A_{1}^{l_{1}^{(4)}} F_{11}^{(4)} \subset V_{2}^{(1)} \backslash G_{31}^{(1)} \Longrightarrow G_{21}^{(3)} \Longrightarrow G_{31}^{(2)} \Longrightarrow G_{41}^{(1)}\right]} \\
& \Longrightarrow\left[G_{11}^{(5)} \Longrightarrow G_{21}^{(4)} \Longrightarrow G_{31}^{(3)} \Longrightarrow G_{41}^{(2)} \Longrightarrow G_{51}^{(1)}\right] \cdots \\
& \left.O^{(1)}\right]
\end{aligned}
$$

Once we substitute $A^{t}$ for $A,[-\pi, \pi)^{n}$ for $E$, and $F_{A^{t}}$, a complete wandering set of $A^{t}$, for $F$ in Theorem 2.3 and on account of Proposition 2.2, we get the following corollary immediately.

Corollary 2.4. Let $A$ be an $n \times n$ real expansive matrix and $E=[-\pi, \pi)^{n}$. Then, there exists $k \in \mathbb{Z}^{n} \backslash\{0\}$ satisfying the property that, given any $\epsilon>0$ with $\mathrm{B}_{\epsilon} \subset E$, there exists an A-dilation wavelet set $G=G_{I} \cup G_{I I}$ such that $G_{I} \subset \mathrm{B}_{\epsilon}$ and $G_{I I} \subset$ $E+2 \pi k$, where $G_{I}$ and $G_{I I}$ are two measurable sets of positive measure.

In the proof of Theorem 2.3 (Corollary 2.4), we can construct for a suitable $k \in \mathbb{Z}^{n} \backslash\{0\}$, a sequence of wavelet sets $G^{(l)}=G_{I}^{(l)} \cup G_{I I}^{(l)}$ such that $G_{I}^{(l)} \subset \mathrm{B}_{\epsilon_{l}}$ and $G_{I I}^{(l)} \subset E+2 \pi k$ for $l=1,2, \ldots$ If $\lim _{l \rightarrow \infty} \epsilon_{l}=0$, then $\lim _{l \rightarrow \infty} m\left(G_{I}^{(l)}\right)=0$ and $G^{(l)} \longrightarrow E+2 \pi k$ as $l \longrightarrow \infty$ in the sense that the measure of their symmetric difference goes to 0 as $l \rightarrow \infty$. But $E+2 \pi k$ is not a wavelet set in general. For instance, in $\mathbb{R}^{1}$, for the dyadic dilation $A=2$, we know that no interval $[-\pi, \pi)+$ $2 \pi k, k \neq 0$, can be a wavelet set since it is not a 2 -dilation tile of $\mathbb{R}$.

The following theorem gives a rough estimate for how close the $k$ chosen in Corollary 2.4 can be to the origin 0 . 
Theorem 2.5. Let $A$ be an $n \times n$ real expansive matrix, and let $A^{t}=T^{-1} C T$ for two real invertible matrices $T$ and $C$ with $\left\|C^{-1}\right\|<1$ (as in Lemma 2.1). Then, for any $k \in \mathbb{Z}^{n}$ satisfying the inequality

$$
\|T k\|>\frac{\sqrt{n}}{2}\|T\| \frac{1+\left\|C^{-1}\right\|}{1-\left\|C^{-1}\right\|}
$$

there exists an A-dilation wavelet set $G=G_{I} \cup G_{I I}$ with $G_{I} \subset \mathrm{B}_{\epsilon}$ and $G_{I I} \subset$ $E+2 \pi k$, where $E=[-\pi, \pi)^{n}$ and $\epsilon>0$ is prefixed.

Proof. Examining the proof of Theorem 2.3, to construct a cube-approximating wavelet set we only need to choose $c \in F_{A^{t}}^{\circ}, m \in \mathbb{Z}, k \in \mathbb{Z}^{n} \backslash\{0\}$, and $\alpha_{1}>0$ such that the five conditions in (2.2) are all satisfied. Then, the existence of a wavelet set $G=G_{I} \cup G_{I I}$ is guaranteed by the construction in the proof of Theorem 2.3.

To satisfy (i) $\left(A^{t}\right)^{-m} E \subset \mathrm{B}_{\alpha_{1}}$, we can require

$$
\sup \left\{\left\|\left(A^{t}\right)^{-m} x\right\|: x \in E\right\} \leq\left\|A^{-1}\right\|^{m} \sqrt{n} \pi:=\alpha_{1}
$$

with $m \geq 0$ to be determined later. To satisfy (ii) $c=2 \pi\left(A^{t}\right)^{-m} k$, and (iv) $\mathrm{B}_{\alpha_{1}}+c \subset F_{A^{t}}$, we can require

$$
\mathrm{B}_{\alpha_{1}}+2 \pi\left(A^{t}\right)^{-m} k \subset\left(A^{t}\right)^{1-m} W_{r} \backslash\left(A^{t}\right)^{-m} W_{r}:=F_{A^{t}}
$$

by Lemma 2.1 and Remark 2.1, where $W_{r}=T^{-1} \mathrm{~B}_{r}$, with $m \geq 0, r>0$ to be determined later. That is,

$$
T\left(A^{t}\right)^{m} \mathrm{~B}_{\alpha_{1}}+2 \pi T k \subset C \mathrm{~B}_{r} \backslash \mathrm{B}_{r} .
$$

Since $\left(C^{-1}\right)^{t} C^{-1}$ is orthogonally similar to a diagonal real matrix, we see that $C \mathrm{~B}_{r}$ is a hyper-ellipsoid whose shortest half axis has length $r /\left\|C^{-1}\right\|$. While for each $m \geq 0, T\left(A^{t}\right)^{m} \mathrm{~B}_{\alpha_{1}}$ is a hyper-ellipsoid contained in the ball $\mathrm{B}_{\beta}$, where $\beta=$ $\alpha_{1}\|T\|\|A\|^{m}$. To satisfy (ii) and (iv), it suffices by (2.9) that we require

$$
r \leq 2 \pi\|T k\|-\alpha_{1}\|T\|\|A\|^{m}<2 \pi\|T k\|+\alpha_{1}\|T\|\|A\|^{m}<\frac{r}{\left\|C^{-1}\right\|} .
$$

Taking $\alpha_{1}=\sqrt{n} \pi\left\|A^{-1}\right\|^{m}$ from (2.7) into (2.10), we have

$$
\begin{aligned}
& r \leq 2 \pi\|T k\|-\sqrt{n} \pi\|T\|\left(\|A\|\left\|A^{-1}\right\|\right)^{m} \\
& <2 \pi\|T k\|+\sqrt{n} \pi\|T\|\left(\|A\|\left\|A^{-1}\right\|\right)^{m}<\frac{r}{\left\|C^{-1}\right\|} .
\end{aligned}
$$

Since $\|A\|\left\|A^{-1}\right\| \geq 1$, we take the minimum $m=0$ and $\alpha_{1}=\sqrt{n} \pi$. Then (2.11) becomes

$$
r \leq 2 \pi\|T k\|-\sqrt{n} \pi\|T\|<2 \pi\|T k\|+\sqrt{n} \pi\|T\|<\frac{r}{\left\|C^{-1}\right\|} .
$$

We are ready to see that, when (2.6) is true and if we take $r=2 \pi\|T k\|-\sqrt{n} \pi\|T\|$, (2.12) will be satisfied. Note that (v) $F_{A^{t}} \backslash \mathrm{B}_{\alpha_{1}}(c)$ has positive measure, since the second inequality sign in (2.12) is strict, where $F_{A^{t}}$ is defined in (2.8).

When (2.6) is true, we can take $m=0$ in the previous proof. This is not unexpected. In the proof of Theorem 2.3, if we let $F_{A^{t}}^{*}=\left(A^{t}\right)^{m} F_{A^{t}}$, which is another complete wandering set for $A^{t}$, then we have $G_{i 1}=\left(A^{t}\right)^{l_{i}-m} F_{i 1}^{*}$ and $G_{i 2}=$ $F_{i 2}^{*}$ in $(2.4)$, i.e. $m^{*}=0$. The condition $(2.6)$ by no means gives the maximum possible range of $k$ which admits a cube approximating wavelet set for all real expansive dilations as it is a very rough estimation. In one-dimensional $\mathbb{R}$ for the dyadic dilation $A=2$, the inequality (2.6) reduces to $|k|>\frac{3}{2}$, i.e., $|k| \geq 2$. Note that 
because of the special structure of the wavelet sets $G$ constructed in Corollary 2.4, $|k|$ cannot be equal to 1 for dilation $A=2$. This statement is implied by [HWW1, Proposition 2.3] with $\alpha=\frac{3}{4} \pi$, which states that if $\psi \in L^{2}(\mathbb{R})$ is a 2-dilation wavelet and $|\hat{\psi}|$ has support contained in $S_{\alpha}=\left[-\frac{8}{3} \alpha, 4 \pi-\frac{4}{3} \alpha\right]$ (or supported in $-S_{\alpha}$ ) for $0<\alpha \leq \pi$, then $|\hat{\psi}(\xi)|=0$ for a.e. $\xi \in H_{\alpha}=\left[-\frac{2}{3} \alpha, 2 \pi-\frac{4}{3} \alpha\right]$ (accordingly for a.e. $\left.\xi \in-H_{\alpha}\right)$. In one dimension for dilation $A=d>1,(2.6)$ reduces to $|k|>\frac{d+1}{2(d-1)}$, and if $d>3$, any $k \in \mathbb{Z} \backslash\{0\}$ will admit a bounded wavelet set.

\section{REFERENCES}

[Au] P. Auscher, Solution of two problems on wavelets, J. Geom. Anal. 5(2) (1995), 181-236. MR.1341029 (96g:42016)

[Br] C. G. Broyden, Basic Matrices - An Introduction to Matrix Theory and Practice, The Macmillan Press Ltd, London and Basingstoke, 1975.

[DDGH] X. Dai, Y. Diao, Q. Gu and D. Han, The existence of subspace wavelet sets, J. Comput. Appl. Math. 155 (1) (2003), 83-90. MR1992291

[DLS1] X. Dai, D. R. Larson, and D. M. Speegle, Wavelet sets in $\mathbb{R}^{n}$, J. Fourier Anal. Appl. 3 (4) (1997), 451-456. MR.1468374 (98m:42048)

[DLS2] Wavelet sets in $\mathbb{R}^{n}$ II, AMS Contemporary Mathematics, 216, 1998, pp. 15-40. MR.1614712 (99d:42054)

[FW] X. Fang and X. Wang, Construction of minimally supported frequency wavelets, J. Fourier Anal. Appl. 2 (4) (1996), 315-327. MR1395767 (97d:42030)

[GH] Q. Gu and D. Han, On multiresolution analysis (MRA) wavelet sets in $\mathbb{R}^{n}$, J. Fourier Anal. Appl. 6 (4) (2000), 437-447. MR.1776974 (2001d:42023)

[HWW1] E. Hernández, X. Wang and Guido Weiss, Smoothing minimally supported frequency wavelets. Part I, J. Fourier Anal. Appl. 2 (4) (1996), 329-340. MR1395768 (97h:42015)

[HWW2] _ Smoothing minimally supported frequency wavelets. Part II, J. Fourier Anal. Appl. 3 (1) (1997), 23-41. MR1428814 (98b:42049)

[Me] Y. Meyer, Wavelets and Operators.Translated from the 1990 French original by D. H. Salinger. Cambridge Studies in Advanced Mathematics. 37, Cambridge University Press, Cambridge, 1992. MR1228209 (94f:42001)

Department of Mathematics and Statistics, McMaster University, 1280 Main Street West, Hamilton, Ontario, Canada L8S 4K1

E-mail address: yuxia@math.mcmaster.ca 